\title{
First supernovae in dwarf protogalaxies
}

\author{
E. O. Vasiliev 1,3 , E. I. Vorobyov ${ }^{2,3}$, and Yu. A. Shchekinov ${ }^{4,5}$
}

1 Tartu Observatory, 61602 Tõravere, Estonia

2 Institute for Computational Astrophysics, Saint Mary's University, Halifax, B3H 3C3, Canada e-mail: vorobyov@ap.smu.ca

3 Institute of Physics, Southern Federal University, Stachki Ave. 194, Rostov-on-Don 344090, Russia e-mail: eugstar@mail.ru

4 Department of Physics, Southern Federal University, Sorge St. 5, Rostov-on-Don 344090, Russia e-mail: yus@phys.rsu.ru

5 Special Astrophysical Observatory, Nizhny Arkhyz, Karachai-Cherkessia 369167, Russia

Received 23 March 2008 / Accepted 21 July 2008

\section{ABSTRACT}

\begin{abstract}
Context. This paper is motivated by the recent detection of many extremely metal-deficient $([\mathrm{Fe} / \mathrm{H}]<-3)$ stars in the Milky Way. Aims. We numerically explore the chemical, thermal, and dynamical evolution of a shell formed by a high-energy supernova explosion $\left(10^{53} \mathrm{erg}\right.$ ) in dwarf protogalaxies with a total (dark matter plus baryonic) mass $10^{7} M_{\odot}$ at a redshift $z=12$. We consider two initial configurations for the baryonic matter, one without rotation and the other having the ratio of rotational to gravitational energy $\beta=0.17$. The (non-rotating) dark matter halo is described by a quasi-isothermal sphere. This choice is motivated by recently proposed mechanisms for rapid flattening of a central cuspy region in dwarf protogalaxies.

Methods. We use a finite-difference numerical hydrodynamics code to simulate supernova explosions in dwarf protogalaxies with axial symmetry. The advection is treated using a third-order piecewise parabolic scheme. The heating and cooling processes in the gas are taken into account by solving the rate equations numerically for the main atomic, molecular, and ionic species in the primordial gas.

Results. We find that the dynamics of the shell is different in protogalaxies with and those without rotation. For instance, the RayleighTaylor instability in the shell develops faster in protogalaxies without rotation. The fraction of a blown-away baryonic mass is approximately twice as high in models with rotation than in models without rotation. We argue that these differences are caused by different initial gas density profiles in non-rotating and rotating protogalaxies. On the other hand, the chemical evolution of gas in protogalaxies with and without rotation is found to be similar. The relative number densities of molecular hydrogen and HD molecules in the cold gas $\left(T \leq 10^{3} \mathrm{~K}\right)$ saturate at typical values of $10^{-3}$ and $10^{-7}$, respectively. The saturation times in models with rotation are somewhat longer than in models without rotation. The clumps formed in the fragmented shell move with velocities that are at least twice as high as the escape velocity. The mass of the clumps is $\sim 0.1-10 M_{\odot}$, which is lower than the Jeans mass. We conclude that the clumps are pressure supported.

Conclusions. A supernova explosion with energy $10^{53}$ erg destroys our model protogalaxy. The clumps formed in the fragmented shell are pressure supported. We conclude that protogalaxies with a total mass $\sim 10^{7} M_{\odot}$ are unlikely to form stars due to high-energy supernova explosions of the first stars.
\end{abstract}

Key words. cosmology: early Universe - galaxies: formation - ISM: molecules - stars: formation - shock waves

\section{Introduction}

The detection of extremely metal-poor stars in our Galaxy with an iron abundance equal to or less than $10^{-3}$ of the solar value (Beers et al. 1992; Christlieb et al. 2002) has motivated scientists to put forward possible scenarios for the formation of such stars. According to Tsujumoto et al. (1999), extremely metalpoor (EMP) stars form in a dense shell produced by type II supernova explosions of the first stars and accrete metals from the surrounding medium during the subsequent evolution. The formation of EMP stars is made possible by fragmentation of the primordial gas in a supernova shell due to efficient cooling by molecular hydrogen and HD molecules. Indeed, the formation of molecular hydrogen increases significantly in the wake of a strong shock wave (Suchkov et al. 1983; Shapiro \& Kang 1987). Moreover, at temperatures lower than $150 \mathrm{~K}$, HD molecules become a much more efficient coolant than molecular hydrogen (e.g., Shchekinov 1986).
Subsequent studies by many authors (see review by Nishi \& Susa 1999; Salvaterra et al. 2004; Machida et al. 2005; Greif et al. 2007) have shown that the likelihood for the formation of EMP stars in supernova-driven shells is sensitive to both the mass of the dark matter halo $\left(M_{\mathrm{h}}\right)$ in a primordial galaxy and the assumed type of supernova explosions. For instance, Nishi \& Susa (1999) have shown that the shell fragmentation due to type II supernova explosions $\left(10^{51} \mathrm{erg}\right)$ is possible only in dark matter haloes with total mass $M_{\mathrm{h}} \gtrsim$ a few $\times 10^{7} M_{\odot}$. Galaxies with $M_{\mathrm{h}} \lesssim 10^{7} M_{\odot}$ may lose its baryonic matter before it has time to fragment and form stars (Ferrara 1998). The likelihood that this blow-away scenario increases if the first stars explode as pair-instability supernovae with the energy release of the order of $10^{53} \mathrm{erg}$ (Bromm et al. 2003; Greif et al. 2007). However, these numerical results were confronted by Machida et al. (2005), who presented a semi-analytic model for the evolution of a gas shell produced by supernova explosions with energy $10^{51}-10^{52}$ erg. They took the $\mathrm{H}_{2}$ and HD chemistry into account and found 
that supernova explosions can induce fragmentation of the gas shell and formation of EMP stars in dark matter haloes with $M_{\mathrm{h}}=\mathrm{a}$ few $\times 10^{6} M_{\odot}$. The typical mass of the fragments in their model is about $1.0 M_{\odot}$, which is consistent with the result by Uehara \& Inutsuka (2000) and similar to the masses of the observed low-metallicity stars (Christlieb et al. 2002).

The likelihood of the formation of EMP stars also depends on the radial distribution of gas in a primordial galaxy prior to supernova explosions. This distribution can (partly) be determined by HII regions around the first stars. For instance, Whalen \& Norman (2004) considered the formation of the HII region in a low-mass dark matter halo with mass $10^{6} M_{\odot}$ and found that the gas is efficiently blown away by a supersonic shock wave accociated with the R-type ionization front. Similar results were obtained by Kitayama et al. (2004) for the same mass of the dark matter halo. They have also considered more massive dark matter haloes and concluded that the HII region is confined within the virial radius in haloes with masses $\gtrsim 10^{7} M_{\odot}$ (the ionization front is of the D-type). More recently, Kitayama \& Yoshida (2005) studied numerically the destruction of dark matter haloes due to the formation of HII regions due to radiation of the first stars and subsequent supernova explosions. Using a one-dimensional Lagrangean hydrodynamics code, they found that the SN shock wave remains well inside the virial radius for dark matter haloes with masses larger than $10^{7} M_{\odot}$. On the other hand, in low-mass dark matter haloes with masses of the order of $10^{6} M_{\odot}$ the shock wave propagates outside the virial radius. These results indicate that the efficiency of halo destruction (and, by implication, the efficiency for the formation of EMP stars) are determined not only by the explosion energy but also by the gas density distribution and radiative feedback from the first stars prior to their explosion.

It now becomes evident that the formation of EMP stars due to fragmentation of supernova driven shells is a complicated phenomenon that depends on a variety of physical conditions in a host protogalaxy, which may vary from allowing star formation to shutting it off completely. In such circumstances, the construction of increasingly more sophisticated numerical models is justified. The majority of abovementioned studies are based on semianalytic models (e.g., Salvaterra et al. 2004; Machida et al. 2005 ) or on one-dimensional numerical hydrodynamic simulations (e.g., Kitayama \& Yoshida 2005). In the framework of multi-dimensional numerical simulations, the priority was often given to a careful investigation of dynamical processes leading to the destruction of a dwarf protogalaxy by supernova explosions, neglecting the chemical processes that control the gas thermal properties. In the present paper, we perform axially symmetric numerical hydrodynamics simulations of high-energy supernova explosions $\left(10^{53} \mathrm{erg}\right)$ in a model dwarf protogalaxy with total (dark matter plus baryonic) mass $1.7 \times 10^{7} M_{\odot}$. We give a careful treatment to the heating and cooling processes and chemical evolution of main molecular, ionic, and atomic species. We seek to determine the effect of galactic rotation on the dynamical and chemical evolution of a supernova-driven shell. The dark matter halo profile in our models is defined by a modified isothermal sphere (as observed in local dwarf galaxies, Burkert 1995), rather than by a cuspy profile (Navarro et al. 1997).

The paper is organized as follows. In Sects. 2 and 3 we describe the model and numerical techniques, respectively; in Sect. 4 we discuss several neglected processes; in Sect. 5 we present results of our numerical simulations. The discussion and conclusions are given in Sects. 6 and 7, respectively. Throughout the paper we assume a $\Lambda$ CDM cosmology with the parameters $\left(\Omega_{0}, \Omega_{\Lambda}, \Omega_{\mathrm{m}}, \Omega_{\mathrm{b}}, h\right)=(1.0,0.76,0.24,0.041,0.73)$ as inferred from the Wilkinson Microwave Anisotropy Probe (WMAP), and deuterium abundance $2.78 \times 10^{-5}$, consistent with the most recent measurements (Spergel et al. 2007).

\section{Model protogalaxy}

Our model protogalaxy consists of a baryonic component surrounded by a spherical dark matter halo. We assume that the dark matter halo profile is spherically symmetric and is determined by a modified isothermal sphere

$\rho_{\mathrm{h}}(r)=\frac{\rho_{0}}{1+\left(r / r_{0}\right)^{2}}$,

where $r_{0}$ and $\rho_{0}$ are the core radius and central density, respectively. Cosmological $N$-body simulations suggest that dark matter haloes (upon their formation) have radial profiles that are cuspy in the central regions and scale as $r^{-3}$ at large radii (Navarro et al. 1997). On the other hand, modelling of the rotation curves in nearby dwarf galaxies indicates that the dark matter profiles have a flat central region and a tail that scales as $r^{-2}$ (Burkert 1995). It is poorly known how and when the transition from cuspy to flat dark matter haloes occurs. Several possible mechanisms that can facilitate transformation of the profile include random velocities of baryons due to a star formation feedback (Mashchenko et al. 2006), initial background perturbations of the dark matter (Mikheeva et al. 2007) and angular momentum transfer from baryons to the dark matter (Tonini et al. 2006). In particular, Mashchenko et al. (2006) suggest that random bulk motions of gas in small primordial galaxies can flatten the central dark matter cusp on relatively short timescales, $\sim 10^{8} \mathrm{yrs}^{1}$. Motivated by this finding, we assume that dark matter haloes in protogalaxies at $z=12$ have already flattened and attained a configuration described by Eq. (1).

We calculate $r_{0}$ and $\rho_{0}$ as a function of the halo mass based on the empirical profile for nearby dwarf galaxies (Burkert 1995; Silich \& Tenorio-Tagle 2001) extrapolated to the early universe by (Fujita et al. 2003)

$r_{0}=8.9 \times 10^{-6} \mathrm{kpc} M_{\mathrm{h}}^{1 / 2} h^{1 / 2} \Omega_{\mathrm{m}}^{-1 / 3}(1+z)^{-1}$,

$\rho_{0}=6.3 \times 10^{10} M_{\odot} \mathrm{kpc}^{-3} M_{\mathrm{h}}^{-1 / 3} h^{-1 / 3} \Omega_{\mathrm{m}}(1+z)^{3}$.

The dark halo mass $\left(M_{\mathrm{h}}\right)$ in our numerical simulations is set to $10^{7} M_{\odot}$, which, at a redshift of $z=12$, corresponds to $3 \sigma$ perturbations in the $\Lambda \mathrm{CDM}$ model for the parameters determined from the third year WMAP data. According to Tegmark et al. (1997) and Shchekinov \& Vasiliev (2006), protogalaxies with dark matter haloes of the order of $10^{7} M_{\odot}$ are expected to cool down efficiently. The virial radius of our model protogalaxy is $r_{\mathrm{v}}=520 \mathrm{pc}$ (for relations between the virial parameters see e.g., Ciardi \& Ferrara 2004).

The initial distribution of the total gas density $\left(\rho_{\mathrm{g}}\right)$ is found by solving numerically the steady-state momentum equations in cylindrical coordinates $(z, r)$

$\frac{u_{\phi}^{2}}{r}=\frac{1}{\rho_{\mathrm{g}}} \frac{\partial p}{\partial r}+\frac{\partial \Phi_{\mathrm{h}}}{\partial r}$,

$\frac{1}{\rho} \frac{\partial p}{\partial z}=-\frac{\partial \Phi_{\mathrm{h}}}{\partial z}$,

${ }^{1}$ For the adopted cosmological model, this timescale corresponds to $1 / 4$ of the comoving age of the universe at $z=12$. 
where $p=\rho_{\mathrm{g}} c_{\mathrm{s}}^{2}$ is the gas pressure, $c_{\mathrm{s}}^{2}=R T / \mu$ is the sound speed, $\mu$ is the mean molecular weight, $\Phi_{\mathrm{h}}$ is the gravitational potential of the dark matter halo, and $u_{\phi}$ is the rotation (azimuthal) velocity of gas. The initial value of $\mu$ is set to 1.22 , which corresponds to a gas of neutral hydrogen and helium with abundances by mass $X=0.76$ and $Y=0.24$, respectively. The gas is assumed to be initially isothermal at a virial temperature $T_{\text {vir }}=5900 \mathrm{~K}$.

In order to solve Eqs. (4) and (5) for the initial gas density distribution, we have to know the initial rotation velocity of gas, $u_{\phi 0}$. We determine $u_{\phi 0}$ in two steps. First, we calculate the circular velocity of gas $u_{\text {circ }}$, which is determined exclusively by the gravitational potential of the dark matter halo (neglecting the contribution from gas pressure gradients)

$u_{\text {circ }}^{2}=r \frac{\partial \Phi_{\mathrm{h}}}{\partial r}$.

Then we introduce a parameter $\alpha<1$, which measures the relative input of rotation to the total support against gravity. The rotation velocity is finally determined as $u_{\phi 0}=\alpha u_{\text {circ }}$. In the following text, we consider two models: model 1 without rotation and model 2 with rotation, for which we choose $\alpha=0.4$. The resulted total rotational energy inside the virial radius is $E_{\mathrm{rot}}=\int u_{\phi 0}^{2} \rho \mathrm{d} V \approx 0.31 \times 10^{51} \mathrm{erg}$. The corresponding ratio $\beta$ of rotational to gravitational energy is equal to 0.17 . We note that $\beta \simeq \alpha^{2}$ because $E_{\text {rot }} / E_{\mathrm{gr}} \simeq u_{\phi}^{2} / u_{\text {circ }}^{2}$, where $E_{\mathrm{gr}}$ is the gravitational energy. We note that our model galaxy has a modest amount of rotation and most support against gravity comes from gas pressure gradients.

The numerical procedure for solving the steady-state Eqs. (4) and (5) to obtain the equilibrium gas density distribution is given in Vorobyov et al. (2004). There is, however, one important difference. The total masses of gas and dark matter halo in protogalaxies are linked through the relation $M_{\mathrm{g}}=M_{\mathrm{h}}\left(\Omega_{\mathrm{b}} / \Omega_{\mathrm{m}}\right)$. In order to satisfy this relation, we iterate solutions until the resulted gas density distribution has attained the mass of about $1.7 \times 10^{6} M_{\odot}$ inside the virial radius.

Once the equilibrium gas density distribution is constructed, we let our model galaxies evolve out of equilibrium. The inner densest regions are characterized by largest cooling rates, and they cool and contract on much shorter time scales than the outer regions. We stop this process when the temperature in the centre drops below $500 \mathrm{~K}$, correspondingly the density increases about 50 times in comparison with the central equilibrium value. This happens at $t \gtrsim 1.0 \mathrm{Myr}$ in model 1 and $t \gtrsim 1.4 \mathrm{Myr}$ in model 2 . The resulted distributions of gas density (top panel), temperature (middle panel), and infall velocity (bottom panel) in model 1 (solid line) and model 2 (dashed and dotted lines) are shown in Fig. 1. More specifically, the dashed and dotted lines show the radial and vertical profiles in model 2 , respectively. We note that in the non-rotating model 1 the distributions are identical in all directions.

The infall velocities in Fig. 1 indicate that only the inner regions of our model galaxy $r \lesssim 50 \mathrm{pc}$ are driven out of equilibrium, while the rest of the galaxy is intact. This out-ofequilibrium region remains confined to the inner $100 \mathrm{pc}$, even if we extend our numerical integration for another several million years. As a result, the radial gas density profiles in model 1 and 2 are distinct throughout the bulk of the galaxy. In particular, the radial gas density profile in model 2 (with rotation) is shallower $\left(\propto r^{-1.7}\right)$ than in model 1 without rotation $\left(\propto r^{-2.0}\right)$, except for the innermost region. The difference in the central gas density between the models with and without rotation can reach a factor of three. However, the masses enclosed inside the virial radius

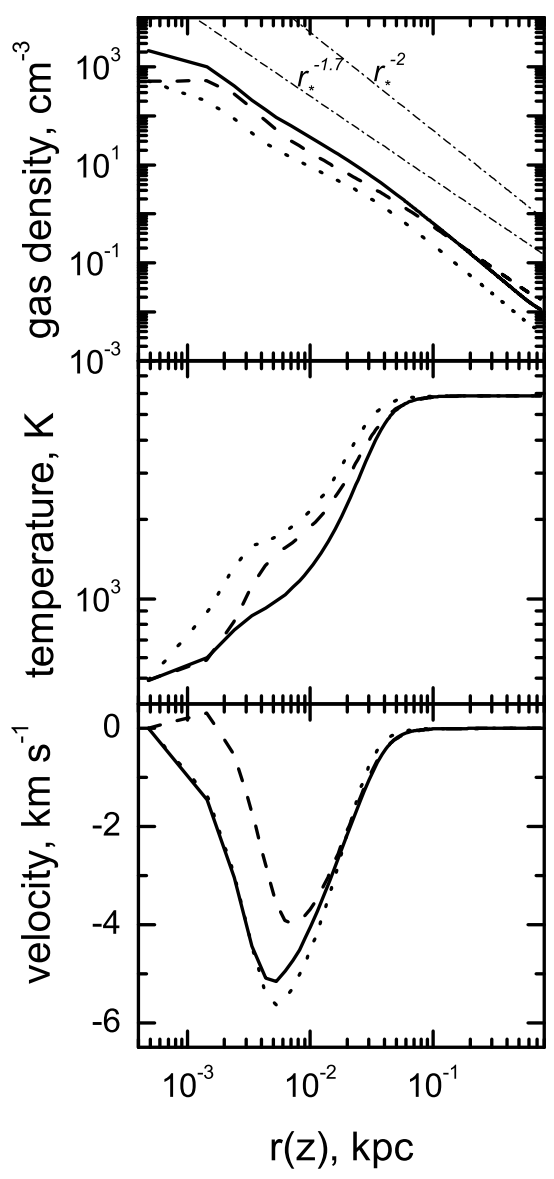

Fig. 1. Profiles for the gas density (top panel), temperature (middle panel), and infall velocity (bottom panel) in the non-rotation model 1 (solid line) and rotating model 2 (dashed and dotted lines). In particular, the dashed and dotted lines show the radial and vertical profiles in model 2 , respectively.

$(\approx 520 \mathrm{pc})$ are approximately the same in both models. The vertical gas distribution in model 2 (dotted line) is characterized by progressively smaller values (at same radii) than in model 1 (solid line). This implies that supernovae remnants in rotating protogalaxies are expected to expand to a larger distance in the vertical direction, which may result in a partial loss of gas by a parent protogalaxy.

Once the central gas temperature drops below $500 \mathrm{~K}$, we release $10^{53} \mathrm{erg}$ of thermal energy in the central sphere with radius $5 \mathrm{pc}$. Such energetic supernovae are expected to result from the pair-instability explosion of massive metal-free stars (Heger \& Woosley 2002). This approach is supposed to reasonably mimic the formation of a first star and its subsequent explosion. The gas temperature in the central sphere is set to $2.5 \times 10^{4} \mathrm{~K}$, typical for HII regions, and gas density is adjusted to maintain the pressure balance between the interior and immediate exterior of the sphere. We assume that this sphere was formed by a collective action of stellar wind and ultraviolet radiation from a massive star (the supernova progenitor) that had resided in the galactic centre before the explosion (we skip this phase in our numerical simulations). Since the physical size of a shock front at initial stages is considerably smaller than our numerical resolution $(\sim 1 \mathrm{pc})$, we allow for the gas to initially evolve adiabatically and switch on cooling only after $6 \times 10^{4}$ yr of evolution. In what follow we will loosely define this hot sphere as a supernova ejecta, although it differs physically from the ejecta in realistic 
supernovae explosions. The initial composition of species inside the sphere corresponds to a fully ionized gas.

\section{Numerical model}

\subsection{Main equations}

The dynamics of the gaseous component in our model protogalaxy is followed by numerically solving a usual set of hydrodynamic equations in cylindrical coordinates $(z, r, \phi)$

$\frac{\partial \rho_{\mathrm{g}}}{\partial t}+\boldsymbol{\nabla} \cdot\left(\rho_{\mathrm{g}} \boldsymbol{u}\right)=0$

$\frac{\partial}{\partial t}\left(\rho_{\mathrm{g}} \boldsymbol{u}\right)+\boldsymbol{\nabla} \cdot\left(\rho_{\mathrm{g}} \boldsymbol{u} \cdot \boldsymbol{u}\right)=-\boldsymbol{\nabla} p-\rho_{\mathrm{g}} \boldsymbol{\nabla} \Phi_{\mathrm{h}}$,

$\frac{\partial \epsilon}{\partial t}+\boldsymbol{\nabla} \cdot(\epsilon \boldsymbol{u})=-p(\boldsymbol{\nabla} \cdot \boldsymbol{u})-\Lambda$,

where $\boldsymbol{u}=\hat{z} u_{z}+\hat{\boldsymbol{r}} u_{r}+\hat{\phi} u_{\phi}$ is the gas velocity in cylindrical coordinates, $\boldsymbol{\nabla}=\hat{z} \partial / \partial z+\hat{\boldsymbol{r}} \partial / \partial r$ is the spatial derivative in cylindrical coordinates modified for the adopted axial symmetry, $\Lambda$ is the cooling rate, and $\epsilon$ is the internal energy density. The hydrodynamic equations are closed by an ideal equation of state $p=(\gamma-1) \epsilon$, where $\gamma$ is set to $5 / 3$. A (small) amount of numerical viscosity is added to the code (according to the usual prescription of von Neumann and Richtmyer) in order to smooth out shock and discontinuities over two computational zones.

Equations (7)-(9) are solved using a finite-difference, operator-split code, which applies the consistent transport technique introduced by (Norman et al. 1980). The code performs well on the standard test suite including the Sedov point explosion test. The advection is treated using a third-order piecewise parabolic advection scheme (Collela \& Woodward 1984). This scheme is known to be more accurate in handling the shocks and contact discontinuities than a commonly used van Leer advecion scheme (see e.g., Stone \& Norman 1992). The computational domain has a size of $750 \mathrm{pc}$ in both the vertical $(z)$ and horizontal $(r)$ directions. Cosmological effects (expansion and bias) are not expected to significantly influence the results on such spatial scales. We assume an equatorial symmetry for simplicity. The numerical resolution is $780 \times 780$ grid zones, which corresponds to a spatial resolution of $\approx 1.0 \mathrm{pc}$ in both coordinate directions. In our simulations self-gravity of the gas is neglected and the dark matter potential is fixed. In a few test runs, we included the self-gravity of the gas but found it dynamically unimportant.

\subsection{Cooling processes and chemical reactions}

The gas component of our model protogalaxy consists of a standard set of species: $\mathrm{H}, \mathrm{He}, \mathrm{H}^{+}, \mathrm{H}^{-}, \mathrm{H}_{2}, \mathrm{H}_{2}^{+}, \mathrm{D}, \mathrm{D}^{+}$, and $\mathrm{HD}$. We also make use of the charge conservation law for electrons. The initial number densities of neutral hydrogen and helium relative to the total number density of gas $n_{\mathrm{g}}=\rho_{\mathrm{g}} /\left(\mu m_{\mathrm{H}}\right)$ are $x[\mathrm{H}]=0.9999 \times 0.76$ and $x[\mathrm{He}]=0.24$, respectively. The initial number densities of ionized hydrogen, molecular hydrogen, and HD molecules relative to $n_{\mathrm{g}}$ are $x\left[\mathrm{H}^{+}\right]=10^{-4}, x\left[\mathrm{H}_{2}\right]=10^{-6}$ and $x[\mathrm{HD}]=10^{-10}$, respectively. The initial relative number density of deuterium is equal to the cosmological value. The relative number densities of the other species in our sample are set initially to a negligible value.

The cooling rates are computed separately for temperatures below and above $2 \times 10^{4} \mathrm{~K}$. In the low-temperature regime, the
Table 1. Chemical reaction rates.

\begin{tabular}{|c|c|}
\hline Reaction & Reference \\
\hline $\mathrm{H}^{+}+\mathrm{e}^{-} \rightarrow \mathrm{H}+\mathrm{h} v$ & Galli \& Palla (1998) \\
\hline $\mathrm{H}+\mathrm{e}^{-} \rightarrow \mathrm{H}^{-}+\mathrm{h} v$ & - \\
\hline $\mathrm{H}^{-}+\mathrm{h} v \rightarrow \mathrm{H}+\mathrm{e}^{-}$ & - \\
\hline $\mathrm{H}^{-}+\mathrm{H} \rightarrow \mathrm{H}_{2}+\mathrm{e}^{-}$ & - \\
\hline $\mathrm{H}^{-}+\mathrm{H}^{+} \rightarrow \mathrm{H}_{2}^{+}+\mathrm{e}^{-}$ & - \\
\hline $\mathrm{H}^{-}+\mathrm{H}^{+} \rightarrow 2 \overline{\mathrm{H}}$ & - \\
\hline $\mathrm{H}^{+}+\mathrm{H} \rightarrow \mathrm{H}_{2}^{+}+\mathrm{h} v$ & - \\
\hline $\mathrm{H}_{2}^{+}+\mathrm{H} \rightarrow \mathrm{H}_{2}+\mathrm{H}^{+}$ & - \\
\hline $\mathrm{H}_{2}^{+}+\mathrm{e}^{-} \rightarrow 2 \mathrm{H}$ & - \\
\hline $\mathrm{H}_{2}+\mathrm{H}^{+} \rightarrow \mathrm{H}_{2}^{+}+\mathrm{H}$ & - \\
\hline $\mathrm{H}_{2}+\mathrm{e}^{-} \rightarrow 2 \mathrm{H}+\mathrm{e}^{-}$ & - \\
\hline $\mathrm{H}_{2}+\mathrm{H} \rightarrow 3 \mathrm{H}$ & MacLow \& Shull (1986) \\
\hline $\mathrm{H}+\mathrm{e}^{-} \rightarrow \mathrm{H}^{+}+2 \mathrm{e}^{-}$ & Abel et al. (1997) \\
\hline $\mathrm{H}^{-}+\mathrm{e}^{-} \rightarrow \mathrm{H}+2 \mathrm{e}^{-}$ & - \\
\hline $\mathrm{H}^{-}+\mathrm{H} \rightarrow 2 \mathrm{H}+\mathrm{e}^{-}$ & Shapiro \& Kang (1987) \\
\hline $\mathrm{D}^{+}+\mathrm{H}_{2} \rightarrow \mathrm{HD}+\mathrm{H}^{+}$ & Galli \& Palla (1998) \\
\hline $\mathrm{H}^{+}+\mathrm{HD} \rightarrow \mathrm{H}_{2}+\mathrm{D}^{+}$ & - \\
\hline
\end{tabular}

cooling rate $\Lambda$ in the energy Eq. (9) includes typical radiative losses in the primordial plasma. Cooling due to recombination and collisional excitation of atomic hydrogen is taken from Cen (1992). It is worth mentioning Galli \& Palla (1998) $\mathrm{H}_{2}$ cooling function almost concides with the total cooling function including $\mathrm{H}-\mathrm{H}_{2}$ and $\mathrm{e}^{-}-\mathrm{H}_{2}$ collisions for $x_{\mathrm{e}}<10^{-4}$ (Glover \& Abel 2008), which is the case in our simulation. Cooling due to molecular hydrogen is taken from Galli \& Palla (1998) and modified for the temperature regime near the CMB radiation temperature (Varshalovich \& Khersonskii 1976; Puy et al. 1993; Le Bourlot et al. 1999). Cooling due to HD molecules is computed according the prescription given in Flower (2000) and Lipovka et al. (2005). In the high-temperature regime, the cooling rates for zero metallicity are taken from Sutherland \& Dopita (1993).

We assume that our atomic, ionic, and molecular species are collisionally coupled to each other and share a common velocity field, which eliminates the need for solving separate equations of motion for each species. Hence, in addition to the usual hydrodynamic Eqs. (7)-(9), we have to solve the continuity and rate equations for the mass densities $\left(\rho_{i}\right)$ of each of the species

$\frac{\partial \rho_{i}}{\partial t}+\boldsymbol{\nabla} \cdot\left(\rho_{i} \boldsymbol{u}\right)=k_{j k} \rho_{j} \rho_{k}-k_{k i} \rho_{k} \rho_{i}$

where the right-hand terms are the sources and sinks due to chemical reactions. The list of reactions and references to the corresponding formation $\left(k_{j k}\right)$ and destruction $\left(k_{k i}\right)$ rates are given in Table 1. We note that we do not solve Eqs. (10) for $\mathrm{D}^{+}$ and $\mathrm{D}$, since the ratio of ionized versus neutral deuterium densities can be implicitly derived from the ratio of ionized versus atomic hydrogen densities (Vasiliev \& Shchekinov 2003). We have also neglected the helium kinetics.

The hydrodynamic Eqs. (7)-(9) and the chemical reaction network (10) are numerically coupled using the following strategy. First, the cooling rate $\Lambda$ is computed and the global hydrodynamic time step $\Delta t_{\mathrm{h}}$ is derived as

$\frac{1}{\Delta t_{\mathrm{h}}}=\frac{1}{\Delta t_{\mathrm{CFL}}}+\frac{1}{\Delta t_{\mathrm{cool}}}$

where $\Delta t_{\mathrm{CFL}}$ is a usual hydrodynamic time step due to the Courant-Friedrichs-Lewy condition and $\Delta t_{\text {cool }}=0.1 \epsilon / \Lambda$ is a characteristic cooling time. Note that $\Delta t_{\mathrm{h}}$ should be minimized over all computational cells.

Once the global hydrodynamic time step is calculated, the solution of Eqs. (7)-(10) proceeds as follows. First, we solve 
the continuity and momentum equations for the total gas density $\rho_{\mathrm{g}}$ (Eqs. (7) and (8), respectively) using a technique described in Sect. 3.1. The solution of the chemical reaction network (10) and energy balance Eq. (9) is split into two parts. The update of internal energy density $(\epsilon)$ and mass densities of each species $\left(\rho_{i}\right)$ due to advection is done by solving the following continuity equations

$$
\begin{aligned}
& \frac{\partial \rho_{i}}{\partial t}+\nabla \cdot\left(\rho_{i} \boldsymbol{u}\right)=0, \\
& \frac{\partial \epsilon}{\partial t}+\boldsymbol{\nabla} \cdot(\epsilon \boldsymbol{u})=0 .
\end{aligned}
$$

The solution technique is exactly the same as for Eq. (7).

The remaining update of $\rho_{i}$ due to chemical reactions is combined with the update of $\epsilon$ due to cooling and compressional heating

$$
\begin{aligned}
& \frac{\partial \rho_{i}}{\partial t}=k_{j k} \rho_{j} \rho_{k}-k_{k i} \rho_{k} \rho_{i}, \\
& \frac{\partial \epsilon}{\partial t}=-p(\boldsymbol{\nabla} \cdot \boldsymbol{u})-\Lambda .
\end{aligned}
$$

To improve the accuracy, we use a predictor-corrector scheme. At each step of the predictor-corrector method, Eqs. (14) are integrated using a fifth-order Runge-Kutta-Carsp method with the adaptive step size control (Press et al. 1992). The global integration time step is determined by $\Delta t_{\mathrm{h}}$. We note that in the hightemperature regime $\left(T>2 \times 10^{4} \mathrm{~K}\right)$ the mass densities of atomic and ionized hydrogen are computed adopting a local thermal equilibrium. More specifically, the ionization and recombination rates for hydrogen are set equal. The mass densities of the other four species $\left(\mathrm{H}^{-}, \mathrm{H}_{2}, \mathrm{H}_{2}^{+}\right.$, and $\left.\mathrm{HD}\right)$ are set to a negligible value.

\section{Neglected processes}

In our numerical simulations, we have neglected several physical processes that may be important in some dwarf protogalaxies. For instance, a massive star - a likely supernova progenitoremits enormous ultraviolet (UV) flux that ionizes the surrounding gas and forms the HII region. The radiative pressure of UV photons from massive stars is so strong that it can blow the gas away from the centre of a low-mass $\left(\sim 10^{6} M_{\odot}\right.$ at $\left.z=20\right)$ protogalaxy (Whalen \& Norman 2004). Our model protogalaxy has a more massive dark matter halo, $M=10^{7} M_{\odot}$, but is located at a lower redshift, $z=12$. An HII region formed inside such a protogalaxy is expected to be confined within $10 \mathrm{pc}$. This estimate is obtained by assuming the emission rate of ionizing photons from the first stars to be $10^{50} \mathrm{~s}^{-1}$ (Schaerer 2002) and background density $n=10 \mathrm{~cm}^{-3}$. It is worth noting however that the size of HII region depends on the dark matter profile of a protogalaxy: it is expected to be larger in protogalaxies with a flat density profiles, and smaller in the cuspy profiles. Nevertheless, in order to avoid an overestimate of the effects of the HII region, we set its size in our numerical simulations to $5.0 \mathrm{pc}$.

Shock waves, another source of ionizing radiation (Shull \& Silk 1979), are efficient only in the adiabatic phase of expansion, when the temperature is much higher than $10^{4} \mathrm{~K}$. They cannot influence significantly the formation of molecules at later times.

Another feedback process that can affect the chemistry of gas is the radiation in the (11.18-13.6) eV band, which photodissociates $\mathrm{H}_{2}$ molecules. Although a photo-dissociative region created by the radiation of a massive star can be quite large (Haiman et al. 1997), the typical relative molecular hydrogen density in this region is quite low, about $10^{-6}$ (taken as an initial value in our numerical simulations). Obviously, the primary source of dissociative photons ceases to exist after the supernova explosion and we expect that the production of dissociative photons by the hot gas of a bubble created by the explosion is negligible. Moreover, $\mathrm{H}_{2}$ molecules cannot be photo-dissociated inside cold and dense regions of a supernova-driven shell due to the self-shielding effect (Draine \& Bertoldi 1996). All this argues against photo-dissociation as an important mechanism for $\mathrm{H}_{2}$ destruction after the supernova explosion.

A positive feedback on the abundance of $\mathrm{H}_{2}$ molecules can come from the X-ray photons produced by supernova explosions (Ferrara 1998; Haiman et al. 1997). However, other coacting mechanisms are likely to be more important. For instance, it is well established that the $\mathrm{H}_{2}$ formation is very efficient behind strong shock waves (Suchkov et al. 1983; Shapiro \& Kang 1987). As a result, the relative density of $\mathrm{H}_{2}$ molecules increases very rapidly below $10^{4} \mathrm{~K}$ and saturates at a so-called "universal" value of about $x\left[\mathrm{H}_{2}\right] \sim(1-2) \times 10^{-3}$ at temperature $\sim 3 \times 10^{3} \mathrm{~K}$ (Susa et al. 1998; Oh \& Haiman 2002). Thus, the X-ray photons have only a minor influence on the $\mathrm{H}_{2}$ abundance in a supernovadriven shell but they can strongly influence the $\mathrm{H}_{2}$ abundance in the gas ahead the shell. However, this is not expected to change the picture as a whole because shock waves created by superovae with the energy release of the order of $10^{53}$ erg are strong.

To summarize, we do not expect that these processes are significant for our model protogalaxy. In a future work, we plan to include radiative transfer in the numerical hydrodynamics simulations to check the validity of our assumptions.

\section{Results}

In this section we perform a comparative study of the long-term evolution of supernova remnants in the non-rotating and mildly rotating galaxies. We note that the equilibrium configuration of gas is not preserved exactly during our numerical simulations. A gradual drift from the initial equilibrium (though very small) introduces a seed perturbation to the gas density, which is necessary for the instabilities to grow. These perturbations can be regarded as initial gas inhomogeneities that are always present in protogalaxies before supernovae explosions.

Figure 2 presents snapshots of the gas density distribution in model 1 (top row) and model 2 (bottom row) at four consecutive times after the supernova explosion. In the non-rotating model 1, a thin shell of compressed gas separating the hot supernova ejecta from the unperturbed gas is clearly seen at $t=1 \mathrm{Myr}$. The shock wave has decelerated by this time and its position coincides with the position of the shell. On the contrary, in the rotating model 2 the shock wave lies ahead of the shell at the same evolutionary time. This is a consequence of a lower gas density in the centre of model 2 (see dashed and dot-dashed lines in Fig. 1). It takes a longer time for the shock wave to decelerate and merge with the shell in model 2 . The shock front in model 2 is profoundly elongated along the vertical axis due to a non-spherical initial distribution of gas.

When the characteristic cooling time becomes shorter than the dynamical time (the age of a supernova remnant), an expanding shell becomes unstable to the Rayleigh-Taylor and KelvinHelmholtz instabilities (Gull 1973). As a result, small ripples that distort a spherical shape of the shell appear in model 1 at $t=1$ Myr. Moreover, because the shell is radiative, we expect that the thermal instability plays a non-negligible role in the formation of the ripples. The subsequent evolution of the shell is governed by the Rayleigh-Taylor instability, which acts mostly 

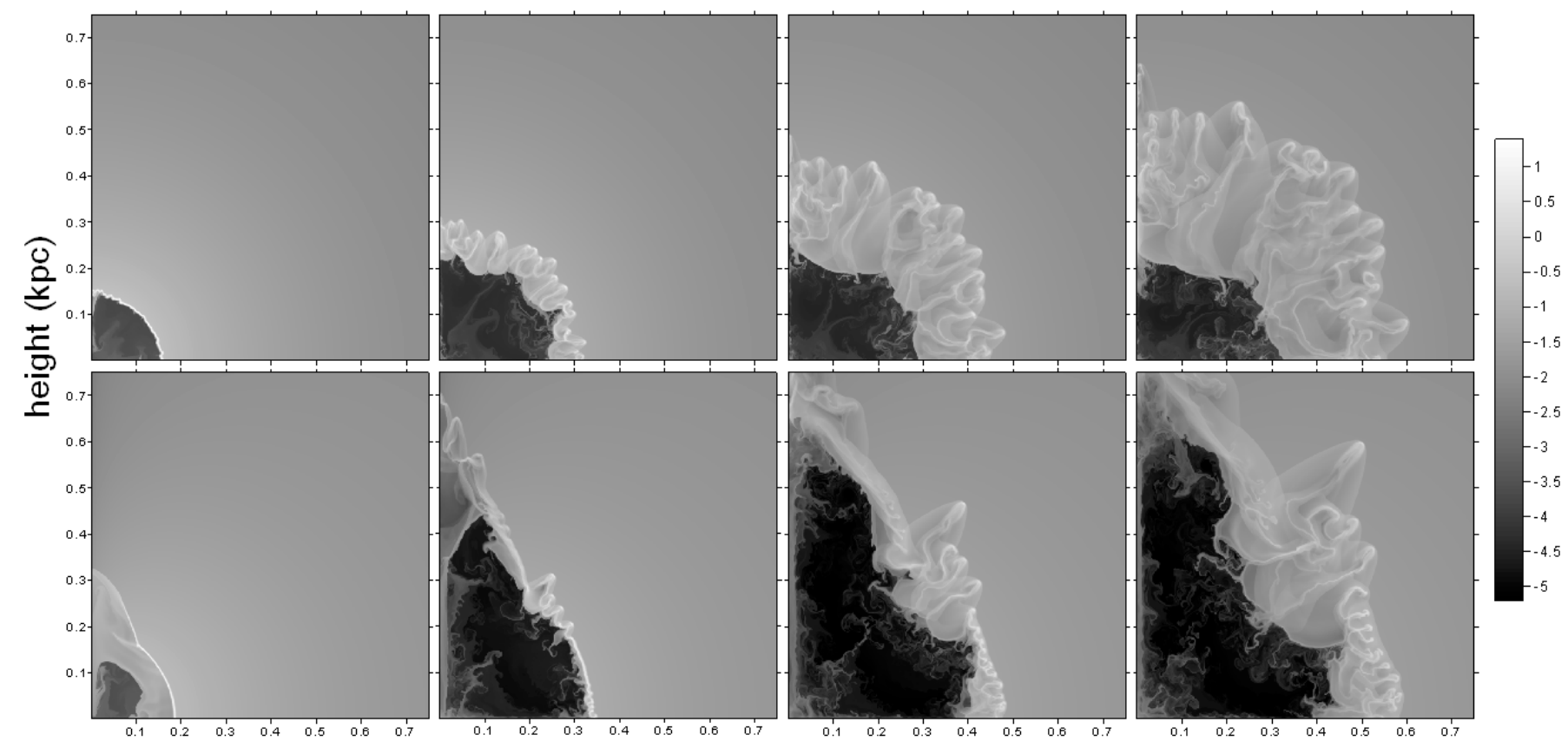

radial distance $(\mathrm{kpc})$

Fig. 2. Logarithmic distribution of density (in $\mathrm{cm}^{-3}$ ) at $t=1,4,8,12 \mathrm{Myr}$ after SN explosion with energy $E_{\mathrm{SN}}=10^{53}$ erg in halo with mass $M=10^{7} M_{\odot}$ for the spin parameter $\beta=0$ - upper and $\beta=0.17$ - low row of panels.

in the compressed gas of the shell outside the interface between hot supernova ejecta and the shell of compressed material. The ejecta itself experiences only large scale distortions, which seem to be inefficient in mixing the metals throughout the shell. The characteristic time for the development of the Rayleigh-Taylor instability is shorter for steeper initial gas density profiles and vice versa. Figure 1 indicates that both the gas density distribution in model 1 and the vertical gas density distribution in model 2 have profiles similar to $r_{*}^{-2}$, where $r_{*}=\left(r^{2}+z^{2}\right)^{1 / 2}$ is the distance from the galactic centre. On the other hand, the radial gas density distribution in model 2 is noticeably shallower and follows an $r_{*}^{-1.7}$ profile. Hence, we expect the Rayleigh-Taylor instability to grow faster in the non-rotating model 1. This is indeed seen in the top row of Fig. 2 - the shell has lost its spherical shape by $t=4 \mathrm{Myr}$ and prominent spurs (or fingers) start to grow into the unperturbed medium. Model 2 shows little spurs at the same evolutionary time, though the shell has already started to show first signs of instability. In the end of numerical simulations, model 1 develops considerably longer spurs than model 2. However, the number of the spurs is smaller in model 1 due to a larger characteristic length of instability. We note that the Rayleigh-Taylor instability is expected to grow faster in protogalaxies with cuspy dark matter haloes due to a steeper gas density profile than in protogalaxies with haloes characterized by a flat central region. However, this effect may be present only in massive protogalaxies (Kitayama \& Yoshida 2005), because steep gas density profiles in low-mass galaxies with cuspy dark matter haloes are likely to be destroyed by ionizing radiation from the first stars.

Figure 2 shows that at $t=12$ Myr some spurs are found outside the virial radius $(520 \mathrm{pc})$ in the both models. This implies that a fraction of the baryonic mass is blown away by the supernova explosion. To calculate this fraction $\left(f_{\text {out }}\right)$, we notice that the spurs are characterized by a systematically lower temperature than the unperturbed medium. We use this property and

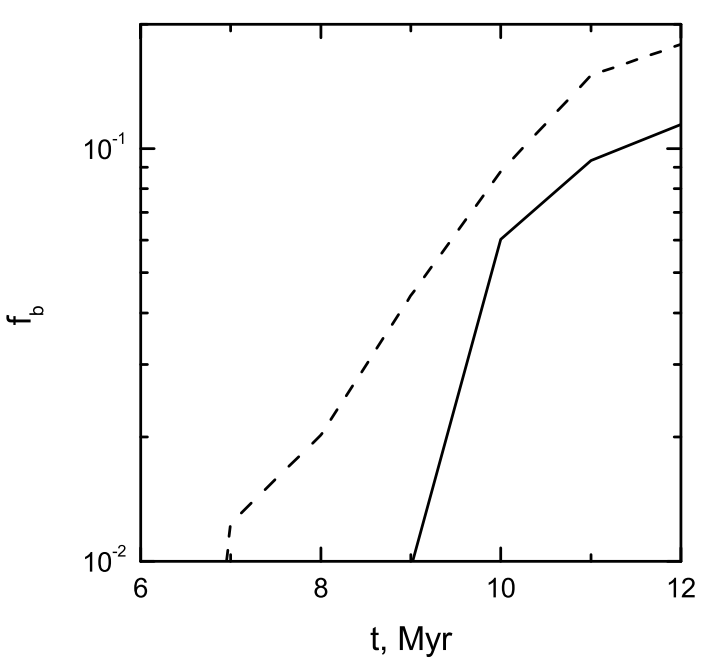

Fig. 3. Fraction of the baryonic mass blown away by a supernova energy release of $10^{53} \mathrm{erg}$ in model 1 (solid) and in model 2 (dash).

find $f_{\text {out }}$ by summing up the gas mass in the computational cells occupied by the spurs at $t=12 \mathrm{Myr}$. We count only those computational cells that lie outside the virial radius. As the spurs cross the virial radius, they sweep up some of the pristine gas. Since we are only interested in the blown-away gas, we subtract the input from this unperturbed gas. The resulted fraction $f_{\text {out }}$ as a function of time is shown in Fig. 3 by the solid (model 1) and dashed (model 2) lines. It is evident that the rotating protogalaxy (model 2) losses roughly twice as much baryonic mass as the non-rotating protogalaxy (model 1). This is a consequence of a shallower initial gas density profile in the rotating protogalaxy.

The spurs have a complicated internal structure. The central regions (or cores) of the spurs are characterized by temperatures lower than $1.5 \times 10^{3} \mathrm{~K}$ and densities roughly ten times 


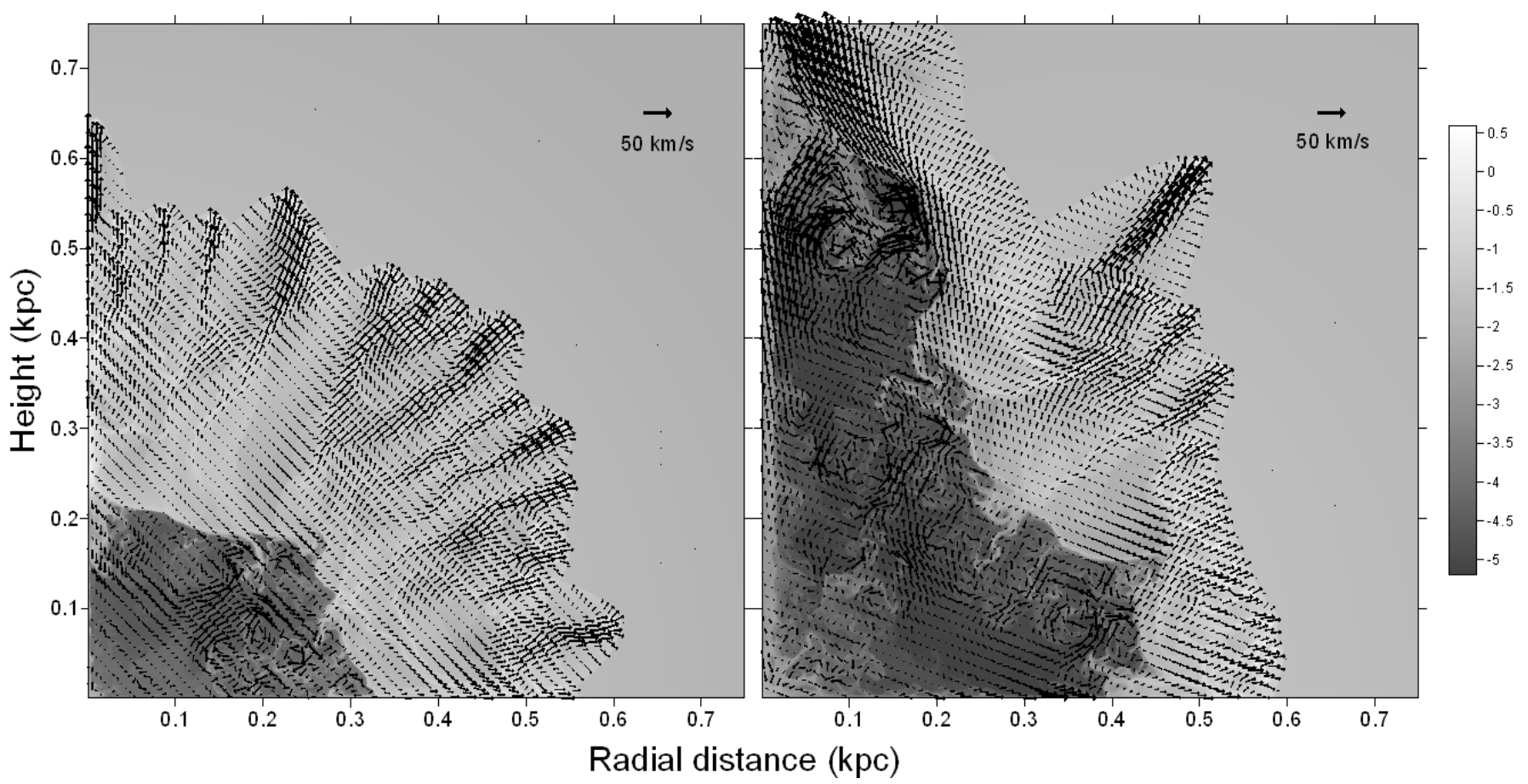

Fig. 4. Velocity field overlaid on the logarithmic density map. Left panel: protogalaxy without rotation, right: with rotation. The arrow in the upper-right corner corresponds to the value of velocity $50 \mathrm{~km} \mathrm{~s}^{-1}$.

higher than those of the neighbouring unperturbed gas. Because the spurs move almost ballistically through the unperturbed gas, bow shocks form around them, creating envelopes of shocked gas. The envelopes are hotter than the cores and are characterized by temperatures about $10^{4} \mathrm{~K}$ and densities roughly four times higher than those of the neighbouring unperturbed gas.

Is the blown-away gas lost to the parent galaxy? Figure 4 shows the gas velocity field superimposed on the gas density distribution at $t=12 \mathrm{Myr}$. We find that the spurs are characterized by mean mass-weighted velocities of the order of $26 \mathrm{~km} \mathrm{~s}^{-1}$ in model 1 and $22 \mathrm{~km} \mathrm{~s}^{-1}$ in model 2 . In the latter model, higher velocities are found near the vertical axis but it may be a numerical artifact due to the axisymmetric nature of our numerical simulations. The spurs appear to have already escaped the galaxy. Indeed, the escape velocity at the virial radius in both models is $v_{\mathrm{e}}=\left(2 g_{\mathrm{r}} r_{\mathrm{v}}\right)^{1 / 2} \approx 13 \mathrm{~km} \mathrm{~s}^{-1}$, where $g_{\mathrm{r}}=G M_{\mathrm{v}}\left(r_{\mathrm{v}}\right) / r_{\mathrm{v}}^{2}$ is the radial gravitational acceleration at the virial radius and $M_{\mathrm{v}}\left(r_{\mathrm{v}}\right)$ is the halo mass inside $r_{\mathrm{v}}$. It is obvious that the mean mass-weighted velocity of the spurs is at least twice as high as the escape velocity at the virial radius, which implies that the spurs might have escaped our protogalaxy.

Figure 5 shows the distribution of gas temperature (left column), relative number densities of molecular hydrogen (middle column) and HD molecules (right column) in the end of numerical simulations at $t=12 \mathrm{Myr}$. The top/bottom rows correspond to model $1 /$ model 2 . It is evident that low gas temperatures (below $10^{3} \mathrm{~K}$ ) are found in the shell and the spurs, where cooling takes place due to $\mathrm{H}_{2}$ and HD molecules. In particular, the lowest temperatures found in the spur cores are of the order of $500 \mathrm{~K}$. The relative number densities of molecular hydrogen and HD molecules in the spur cores are quite large, approximately $10^{-3}$ and $10^{-7}$, respectively. We note that the quoted $\mathrm{H}_{2}$ relative number density is close to a so-called "freeze-out" value (Susa et al. 1998; Oh \& Haiman 2002). A sharp decrease in the $\mathrm{H}_{2}$ relative number density in the envelopes is explained by efficient destruction of $\mathrm{H}_{2}$ molecules due to collisions with hydrogen atoms via the following reaction: $\mathrm{H}_{2}+\mathrm{H} \rightarrow 3 \mathrm{H}$. Shock waves produced by supersonic spurs heat the compressed gas to temperatures above $10^{4} \mathrm{~K}$, at which the collisional destruction of molecular hydrogen becomes dominant. There is no significant difference in the temperatures and relative number densities of molecular species found between the two models. That is not unexpected because strong shock waves lead to similar final temperatures and relative number densities of species in the gas.

Figures 6 and 7 present the temporal evolution of different molecular hydrogen tracers in model 1 and model 2 , respectively. In particular, filled squares show the total molecular hydrogen mass $M_{\mathrm{H}_{2}}$, the filled and open circles yield the total mass of gas with the relative number density of molecular hydrogen $x\left[\mathrm{H}_{2}\right] \geq 5 \times 10^{-4}$ and $x\left[\mathrm{H}_{2}\right] \geq 10^{-3}$, respectively. In addition, we keep track of the gas mass with temperature $\leq 10^{3} \mathrm{~K}$ (filled triangles) and $\leq 500 \mathrm{~K}$ (open trinagles). All molecular hydrogen tracers are calculated inside the computational domain.

The comparison of Figs. 6 and 7 shows that the $\mathrm{H}_{2}$ traces saturate during the evolution. The saturation is explained by the fact that we consider the gas evolution behind strong shock waves, where the $\mathrm{H}_{2}$ relative density rapidly reached a "universal" value $\sim 10^{-3}$ (see Sect. 4). The saturation times in model 2 are systematically longer than in model 1 . This can be attributed to longer cooling times in model 2 due to a shallower initial gas density profile. The filled circles in Figs. 6 and 7 indicate that the total mass of gas with $x\left[\mathrm{H}_{2}\right] \geq 5 \times 10^{-4}$ saturates at $(2-3) \times 10^{5} M_{\odot}$, which corresponds to $\simeq 10 \%$ of the total baryonic mass in our model galaxy. The total gas mass with $x\left[\mathrm{H}_{2}\right] \geq 10^{-3}$ (open circles) saturates at $\sim 10^{5} M_{\odot}$. The gas mass with temperatures below $500 \mathrm{~K}$ approaches an upper limit of $\sim 2 \times 10^{4} M_{\odot}$. The total mass of molecular gas saturates at approximately 300-400 $M_{\odot}$, which is close the estimates made by Ferrara (1998). It is also evident that the $\mathrm{H}_{2}$ tracers in model 2 are characterized by saturated values that are systematically larger than in model 1 . This 

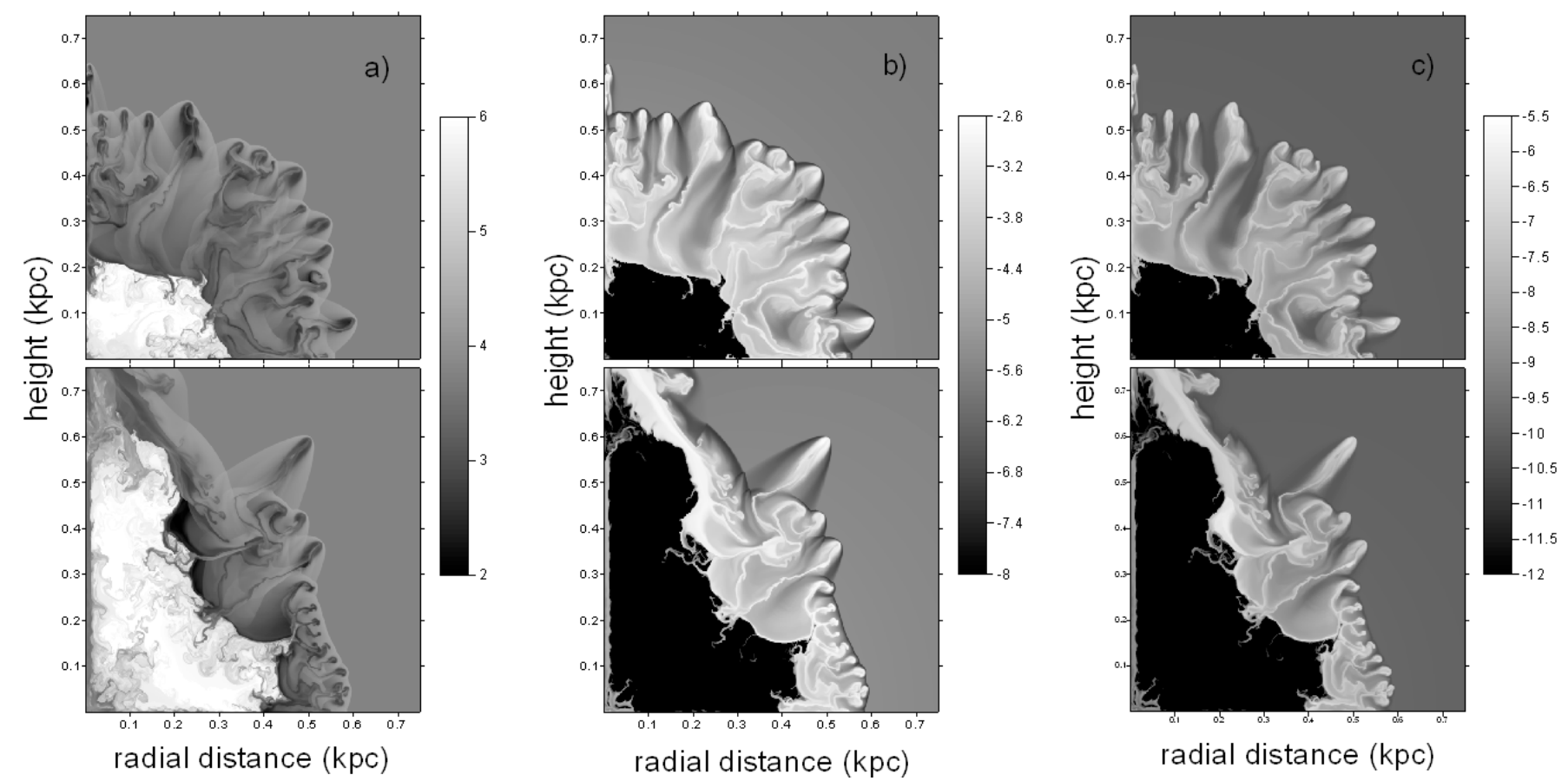

Fig. 5. Logarithmic distribution of temperature (left panel), $\mathrm{H}_{2}$ (middle) and $\mathrm{HD}$ (right) abundances at time $t=12 \mathrm{Myr}$ after the explosion of SN with energy $E_{\mathrm{SN}}=10^{53} \mathrm{erg}$ in halo with mass $M=10^{7} M_{\odot}$ and the parameter $\beta=0$ (upper panels) and $\beta=0.17$ (lower panels).

is due to the fact that the gas mass that crosses the shock wave front is larger in model 2 than in model 1.

\section{Discussion}

We have considered a SN explosion in protogalaxies with and without rotation, and found that rotation changes geometry and dynamics of the flow after the explosion, but does not affect significantly gas chemistry of the final state. Some differences can be found in temporal evolution, however the saturated values of molecular abundances in all considered models in the two cases lie in a very narrow range. It is quite expected that protogalaxies with the ratio of rotational to gravitational energy larger than that in model $2(\beta=0.17)$ are strongly violated in the vertical direction by radiation from the progenitor of a SN. This is basically connected with the fact that the central gas density decreases with the parameter $\beta$ approximately as $n_{\mathrm{c}} \sim \exp (-\beta)$, and the characteristic size of a photo-ionizationally violated region varies as $R \sim n_{\mathrm{c}}^{-2 / 3}$. Moreover, disks in such haloes are more extended in the radial direction with a flat radial density profile

$n \sim r^{-A}$,

where $A \simeq-2 \beta+2$, versus

$n \sim z^{-2}$,

in the vertical direction. This means that photoionizing radiation propagates and evacuates gas mostly perpendicular to the plane around the galactic centre. As a result, when a SN explodes, its shell will expand through this cave, such that a significant fraction of gas can be blown away into the intergalactic medium. One can therefore speculatively conclude that the effects from ionizing photons of the progenitor of a SN will be more pronounced in rotating galaxies in the form of jet-like outflows.

\subsection{Evolution of fragments}

One can see from Figs. 2 and 5 that the typical radial length of the most cold and dense regions is several parsecs. The density of such clumps is about an order of magnitude higher than that of the surrounding gas, whereas the temperature is about an order lower, thus the clumps are in pressure equilibrium with the environment. The clumps form due to desintegration of the shock wave under Rayleigh-Taylor instability which develops when gas behind the shock front starts cooling rapidly and the front decelerates. A typical size of fragments is expected to be close to the thickness of the compresses gas behind the front at the moment, when it becomes unstable.

Among possible mechanisms of cloud destruction stripping of the external layers of clouds seems the most efficient under the conditions of interest. This process operates mostly by Kelvin-Helmholtz instability (e.g., Klein et al. 1994) continuing on shorter scales of a single cloud. The typical stripping time is therefore of the order of Kelvin-Helmholtz instability $t_{\mathrm{KH}}^{-1}=k v / \chi^{1 / 2}$ (Chandrasekhar 1961), where $\chi$ is the density ratio between the cloud and the background medium. For typical parameters $v=20 \mathrm{~km} \mathrm{~s}^{-1}, \chi \sim 10$ and the wavelength of the order of the clump radius $a_{0} \sim 10 \mathrm{pc}$, the destruction time is $\sim 3$ Myr. This is short compared to the dynamical time, and from this point of view dense clumps should be destroyed quickly. However, the radiative cooling time is of the same order $t_{\mathrm{c}} \sim 1-3 \mathrm{Myr}$, which means that the density increase always connected with the radiative cooling can inhibite the destruction through stripping, so that the clumps can survive on longer dynamical time. The clumps however asymptotically are destroyed, which is seen from the fact that the mass contained in relatively dense $\left(n>0.56 \mathrm{~cm}^{-3}\right)$ and cold $\left(T<5 \times 10^{3} \mathrm{~K}\right)$ fragments decreases at $t>3 \mathrm{Myr}$ as shown in Figs. 6 and 7 by dashed lines. Moreover, a typical mass of the most dense $\left(n>1.8 \mathrm{~cm}^{-3}\right)$ clumps $M \sim 0.1-10 M_{\odot}$ (see Fig. 8) is much smaller than the Jeans mass for the corresponding conditions $\left(n \lesssim 1.8 \mathrm{~cm}^{-3}\right.$ and 


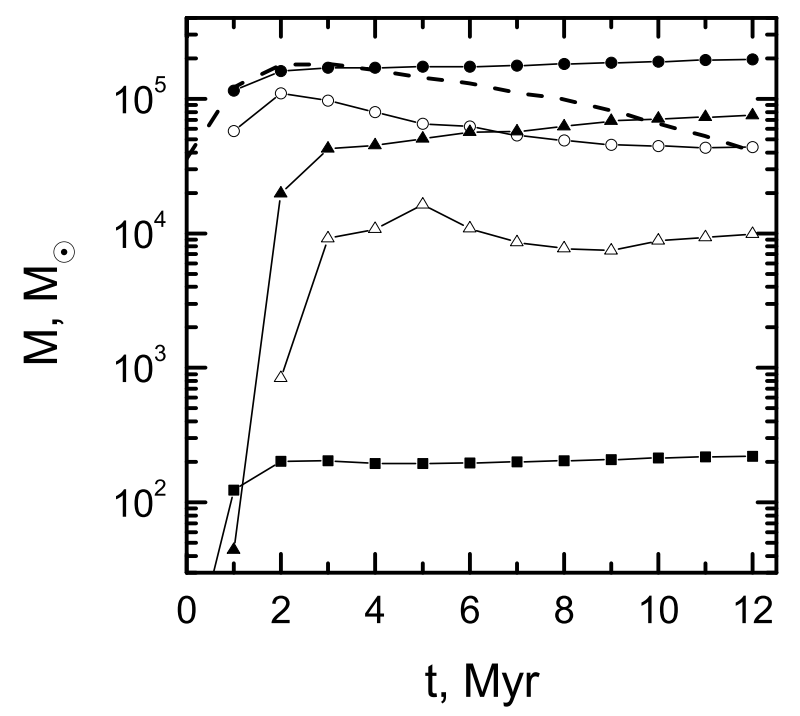

Fig. 6. The total mass of gas in computational domain for model 1 with $\mathrm{H}_{2}$ abundance higher than $x\left[\mathrm{H}_{2}\right]=5 \times 10^{-4}$ (filled circles), $x\left[\mathrm{H}_{2}\right]=10^{-3}$ (open circles), with temperature lower than $T \leq 10^{3} \mathrm{~K}$ (filled triangles), $T \leq 500 \mathrm{~K}$ (open triagles). The line with filled squares represents the total mass of molecular hydrogen. The dashed line shows the gas mass contained in fragments with density $\log n>-0.25$ and temperature $T<5 \times 10^{3} \mathrm{~K}$.

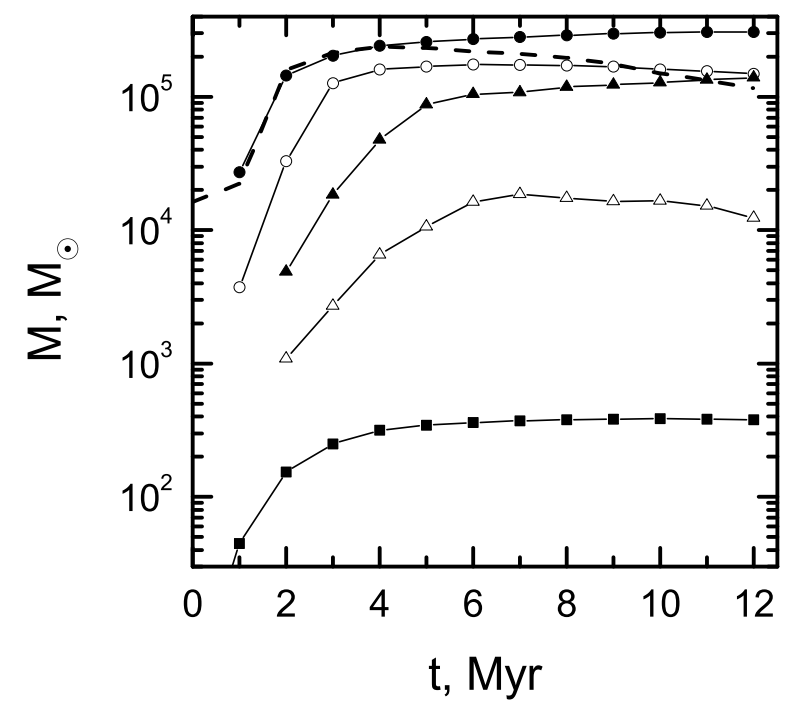

Fig. 7. The same as in Fig. 6 for model 2.

$\left.T \sim 0.5-1 \times 10^{3} \mathrm{~K}\right)$. All this means that protostellar clouds do not form in the shell unless the clumps merge.

In this connection it is worth mentioning the conclusion by Salvaterra et al. (2004) that the shell formed by a SN in the conditions similar to that considered here can fragment only through gravitational instability on to larger masses. One can think that the two instabilities - Kelvin-Helmholtz and gravitational - complement each other, in the sense that while KelvinHelmholtz instability of an expanding SN shell breakes it on to separate fragments (clouds), the gravitational instability stimulates merging of these low-mass fragments, and essentially can result in formation of protostellar clouds of sufficiently large masses. This possibility depends, however, on interelation between the characteristic times of gravitational instability and destruction of the fragments through stripping. As seen from Fig. 4 the relative velocities between the fragments and the ambient gas

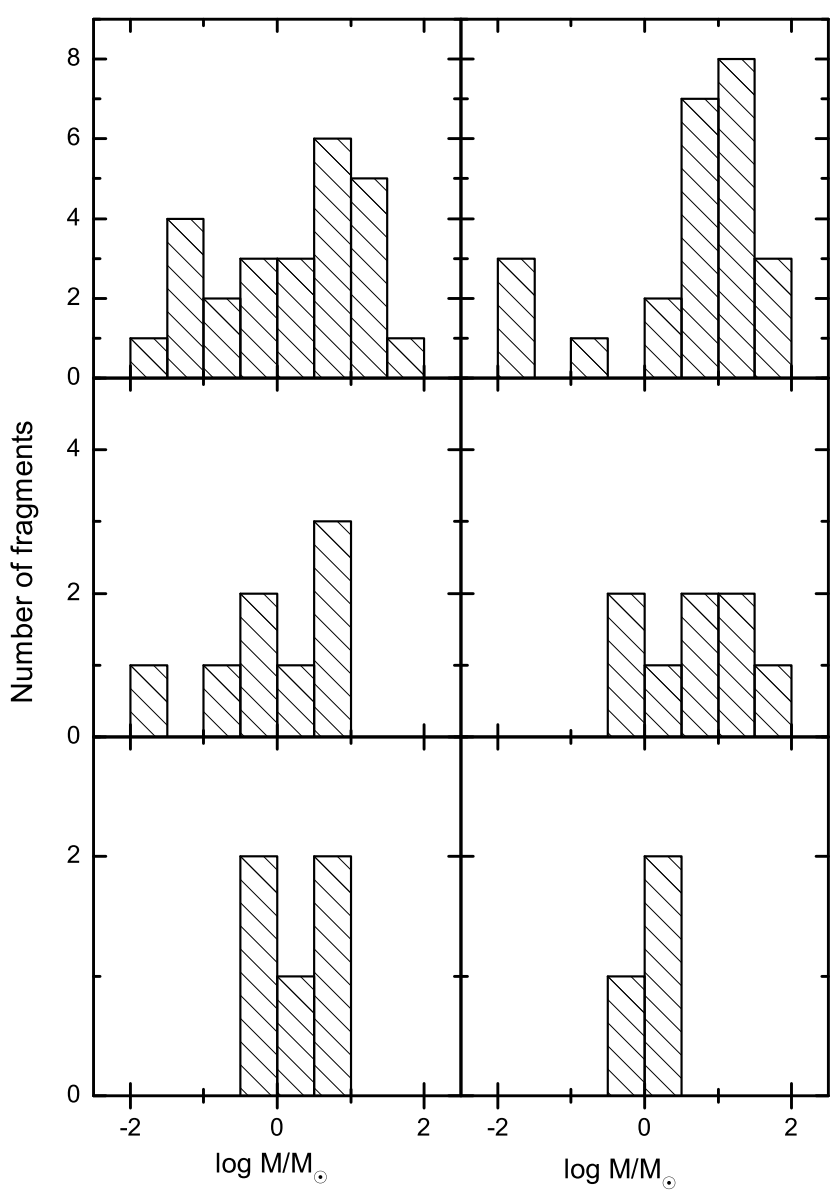

Fig. 8. Number of fragments in the expanding shell at $t=12 \mathrm{Myr}$ for a protogalaxy without (left) and with (right) rotation; panels from the uppermost to the lowermost correspond to the fragments with $\log n>$ $-0.25,0,0.25$, respectively; the mass of the fragments was calculated as $\bar{\rho} S^{3 / 2}$, where $S$ is the area of a filamentary fragment on the "radiusheight" plane, $\bar{\rho}$ is the mean density in it; note, that the fragments of low density do look more filamentary and irregularly shaped than the dense ones, what therefore explains a higher spread of masses of fragments with lower density limit.

remain asymptotically quite high, $20-30 \mathrm{~km} \mathrm{~s}^{-1}$, which results in a relatively short stripping destruction time: $\sim 1 \mathrm{Myr}-$ much shorter than Jeans time. From this point of view the process of star formation in an expanding SN shell meets difficulties: from one side, low-mass fragments originated through disruption of the shell are sub-Jeans and cannot form protostellar condensation, on the other side, destruction of these fragments by stripping prevents them to be gravitationally collected into massive overcritical clouds.

\subsection{Mixing of metals}

Supernovae are recognized as a factory of metals and dust, although many issues related to the efficiency of metal mixing and production of dust particles inside the ejecta, destruction of dust in supernovae shells are still under discussion (Madau et al. 2001; Sugerman et al. 2006; Meikle et al. 2007; Venkatesan et al. 2006). In particular, two conflicting conclusions about the efficiency of dust production in SN 2003gd event by Sugerman et al. (2006) from one side and by Meikle et al. (2007) from the other have to be mentioned. As far as mixing of metals in a SN driven shell is concerned, we believe that metals mostly confined in 
the border between the hot bubble and the surrounding interstellar gas, do penetrate and mix in the swept-up shell very slowly. Mixing of metals in these conditions is determined by RayleighTaylor instability in the interface layer (Wang \& Chevalier 2001) which develops on times longer than the age of the remnant. Indeed, for decelerating shells the criterion for Rayleigh-Taylor instability reads $k<1 / 6 \Delta R$, where $\Delta R$ is the thickness of the shell, $k$ is wavenumber (Vishniac 1983; Vishniac \& Ryu 1989). On the other hand, the instability increment is $\gamma_{\mathrm{RT}} \sim \sqrt{k|a|}$, where $a$ is the deceleration. This gives for a radiative remnant of the age $t$ the restriction $\gamma_{\mathrm{RT}} t<\sqrt{2}$, which means that the perturbations amplitude can grow at most by factor of $\mathrm{e}^{\sqrt{2}}$ (Kas' yanova $\&$ Shchekinov 2005), and therefore, mixing of metals through the whole volume of the swept-up gas seems to be rather inefficient. From the point of view of our simulations this means that the effects of metals on cooling and chemistry of the sweptup gas can be neglected in the initial expansion $t \lesssim 1-3$ Myr. Later on, at $t \lesssim 3 \mathrm{Myr}$, the shell enters an accelerating phase so that Rayleigh-Taylor instability grows faster. However, the interface between the shell and the metal enriched ejecta remains weakly distorted on large spatial scales, and therefore the metals remain mostly locked in a relatively narrow layer around the interface.

\section{Conclusions}

In this paper we have considered numerically the effect of energetic supernovae explosions $\left(10^{53} \mathrm{erg}\right)$ in non-rotating and rotating protogalaxies with the total mass $10^{7} M_{\odot}$ at a redshift of $z=12$. The assumption of axial symmetry allowed us to evolve the supernova driven shell for several tens of million years. Specifically, we find the following.

- The process of the shell destruction is different for nonrotating and rotating protogalaxies. Fingers or spurs formed due to various instabilities (mainly, due to the RayleighTaylor instability) grow faster and can be found on larger distances in non-rotating galaxies. That is explained by a steeper initial gas density profile in non-rotating protogalaxies and, as a consequence, by a faster characteristic timescale for the development of the Rayleigh-Taylor instability.

- The supernova evacuates a significant portion of the initial baryonic mass to radial distances comparable with or larger than the virial radius. At $t=12 \mathrm{Myr}$ after the supernova explosion some of the fragments of the destructed shell have attained mean mass-weighted velocities of the order of $30 \mathrm{~km} \mathrm{~s}^{-1}$ and $25 \mathrm{~km} \mathrm{~s}^{-1}$ in non-rotating and rotating protogalaxies, respectively. These values are at least twice as high as the escape velocity at the virial radius, which implies that the fragments eventually may escape the protogalaxy. We estimate the fraction of this blown-away gas in the end of our numerical simulations to be about $10 \%$ and $20 \%$ of the initial gas mass for models without and with rotation, respectively. The re-collapse phase of the shell sets in faster in models without rotation than in models with rotation. These results are mostly consistent with the intuitive expectations as far as the difference between models with and without rotation is concerned.

- The relative number densities of molecular hydrogen and HD molecules in the fingers and spurs are found to be quite large, approximately $10^{-3}$ and $10^{-7}$, respectively. The typical temperature in the spur cores is of the order of $500 \mathrm{~K}$ at $t \gtrsim 8 \mathrm{Myr}$ after the supernova explosion.
- The total gas mass with $x\left[\mathrm{H}_{2}\right] \geq 10^{-3}$ saturates at $2 \times 10^{5} M_{\odot}$, which corresponds to approximately $10 \%$ of the total baryonic mass in our model galaxy. The corresponding total mass of molecular hydrogen is about 300-400 $M_{\odot}$. The saturation time-scales in the model with rotation are systematically longer than in the model without rotation. This can be attributed to longer characteristic cooling times in the model with rotation due to its shallower initial gas density profile.

Finally, we would like to note that the typical masses of most fragments (assuming their spherical symmetry) are $\sim 0.1-10 M_{\odot}$. For the typical densities and temperatures in the fragments to be $\sim 0.5-1 \mathrm{~cm}^{-3}$ and $(0.5-1) \times 10^{3} \mathrm{~K}$, respectively, these masses are strongly sub-Jeans and the fragments are expected to be pressure-supported. Their further evolution depends on both the efficiency of cooling and destruction due to the KelvinHelmholtz instability. We do not expect that the low-mass stars can be formed in such conditions as suggested by MacKey et al. (2003), and Salvaterra et al. (2004). In our opinion, a more feasible mechanism for low-mass, metal-poor star formation is related with the re-collapse of a supernova bubble in protogalaxies, whose total gravitational binding energy is much larger than supernova energy.

Acknowledgements. We acknowledge critical remarks by the anonymous referee. We acknowledge Eugene Matvienko for his program of statistical processing. This work is supported by the RFBR (project codes 06-02-16819 and 0802-91321). E.O.V. and Yu.A.S. acknowledge partial support from the Federal Agency of Education (project code RNP 2.1.1.3483) and Rosnauka Agency grant No 02.438.11.7001. E. O. V. is supported by the RFBR through the mobility programme grant 08-02-90706. The simulations were done on the Shared Hierarchical Academic Research Computing Network (SHARCNET) while E.I.V. was a CITA National Fellow at the University of Western Ontario.

\section{References}

Abel, T., Anninos, P., Zhang, Yu., \& Norman, M. L. 1997, NewA, 2, 181 Abel, T., Bryan, G. L., \& Norman, M. L. 2002, Science, 295, 93 Beers, T., Preston, G., \& Shectman, S. 1992, AJ, 103, 1987 Bromm, V., \& Loeb, A. 2003, Nature, 425, 812 Bromm, V., Yoshida, N., \& Hernquist, L. 2003, ApJ, 596, L135 Burkert, A. 1995, ApJ, 447, L25

Cen, R. 1992, ApJ, 78, 341

Ciardi, B., \& Ferrara, A. 1997, ApJ, 483, L5

Ciardi, B., \& Ferrara, A. 2005, Space Sci. Rev., 116, 625

Chandrasekhar, S., 1961 Hydrodynamic and Hydromagnetic Stability (NY: Dover)

Christlieb, N., Bessel, M. S., Beers, T. C., et al. 2002, Nature, 419, 904

Collela, P., \& Woodward, P. R. 1984, J. Comp. Phys., 54, 174

Draine, B. T., \& Bertoldi, F. 1996, ApJ, 468, 269

Ferrara, A. 1998, ApJ, 499, L17

Ferrara, A., Pettini, M., \& Shchekinov, Yu. A. 2000, MNRAS, 319, 539

Flower, D. 2000, MNRAS, 318, 875

Fujita, A., Martin, C. L., Mac Low, M.-M., \& Abel, T. 2003, ApJ, 599, 50

Galli, D., \& Palla, F. 1998, A\&A, 335, 403

Glover, S. C. O., \& Abel, T. 2008 [arXiv:0803. 1768]

Greif, T. H., Johnson, J. L., Bromm, V., \& Klessen, R. S. 2007, ApJ, 670, 1

Gull, S. F. 1973, MNRAS, 161, 47

Haiman, Z., Rees, M. J., \& Loeb, A. 1997, ApJ, 476, 458

Heger, A., \& Woosley, S. E. 2002, ApJ, 567, 532

Kas'yanova, M. V., \& Shchekinov, Yu. A. 2005, Astr. Rep., 49, 863

Kitayama, T., \& Yoshida, N. 2005, ApJ, 630, 675

Kitayama, T., Yoshida, N., Susa, H., \& Umemura, M. 2004, ApJ, 613, 631

Klein, R. I., McKee, C. F., \& Colella, P. 1994, ApJ, 420, 213

Le Bourlot, J., Pineau des Forets, G., \& Flower, D. 1999, MNRAS, 305, 802

Lipovka, A., Núñez-López, R., \& Avila-Reese, V. 2005, MNRAS, 361, 850

Machida, M. N., Tomisaka, K., Nakamura, F., \& Fujimoto, M. Y. 2005, ApJ, 622,39

MacKey, J., Bromm, V., \& Hernquist, L. 2003, ApJ, 586, 1

MacLow, M.-M., \& Shull, J. M. 1986, ApJ, 302, 585

MacLow, M.-M., \& Ferrara, A. 1999, ApJ, 513, 142 
Madau, P., Ferrara, A., \& Rees, M. J. 2001, ApJ 555, 92

Maiolino, R., Schneider, R., Oliva, E., et al. 2004, Nature, 431, 533

Mashchenko, S., Couchman, H. M. P., \& Wadsley, J. 2006, Nature, 442, 539

Meikle, W. P. S., Mattila, S., Pastorello, A., et al. 2007, ApJ, 665, 608

Mikheeva, E. V., Doroshkevich, A. G., \& Lukash, V. N. 2007 [arXiv:0712.1688]

Mori, M., Ferrara, A., \& Madau, P. 2002, ApJ, 571, 40

Navarro, J. F., Frenk, C. S., \& White, S. D. M. 1997, ApJ, 490, 493

Nishi, R., \& Susa, H. 1999, ApJ, 523, L103

Norman, M. L., Wilson, J. R., \& Barton, R. 1980, ApJ, 239, 968

Oh, S. P., \& Haiman, Z. 2002, ApJ, 569, 558

Press, W. H., Teukolsky, S. A., Vetterling, W. T., \& Flannery, B. P. 1992, Numerical Recipes in FORTRAN, 2nd edn (Cambridge: Cambridge Univ. Press)

Puy, D., Alecian, G., Le Bourlot, J., Leorat, J., \& Pineau Des Forets, G. 1993, A\&A, 267, 337

Salvaterra, R., Ferrara, A., \& Schneider, R. 2004, NewA, 10, 113

Schaerer, D. 2002, A\&A, 382, 28

Shapiro, P. R., \& Kang, H. 1987, ApJ, 318, 32

Shchekinov, Yu. A. 1986, SovA Lett., 12, 211

Shchekinov, Yu. A., \& Vasiliev, E. O. 2006, MNRAS, 368, 454
Shull, J. M. \& Silk J., 1979, ApJ, 234, 427

Silich, S., \& Tenorio-Tagle, G. 2001, ApJ, 552, 91

Spergel, D. N., Bean, R., Doré, O., et al. 2007, ApJS, 170, 377

Stone, J. M., \& Norman, M. L. 1992, ApJS, 80, 753

Suchkov, A. A., Shchekinov, Yu. A., \& Edelman, M. A. 1983, Astrophysics, 18, 360

Sugerman, B. E. K., Ercolano, B., Barlow, M. J., et al. 2006, Science, 313, 196 Susa, H., Uehara, H., Nishi, R., \& Yamada, M. 1998, Prog. Theor. Phys., 100, 63 Sutherland, R. S., \& Dopita, M. A. 1993, ApJS, 88, 253

Tegmark, M., Silk, J., Rees, M. J., et al. 1997, ApJ, 474, 1

Tonini, C., Lapi, A., \& Salucci, P. 2006, ApJ, 649, 591

Tsujimoto, T., Shigeyama, T., \& Yoshii, Y. 1999, ApJ, 519, L63

Uehara, H., \& Inutsuka, S. 2000, ApJ, 531, L91

Varshalovich, D. A., \& Khersonskii, V. K. 1976, Sov. Astron. Lett., 2, 227

Vasiliev, E. O., \& Shchekinov, Yu. A. 2003, Astron. Rep., 47, 979

Venkatesan, A., Nath, B. B., \& Shull, J. M. 2006, ApJ, 640, 31

Vishniac, E. T. 1983, ApJ, 274, 152

Vishniac, E. T., \& Ryu, D. 1989, ApJ, 337, 817

Vorobyov, E. I., Klein, U., Shchekinov, Yu. A., \& Ott, J. 2004, A\&A, 413, 939

Wang, Ch.-Y., \& Chevalier, R. A. 2001, ApJ, 549, 1119

Whalen, D., Abel, T., \& Norman, M. L., 2004, ApJ, 610, 14 